\title{
Two-Dimensional Analysis of Electrokinetically Driven Out-of-Plane Vortices in a Microchannel Liquid Flow Using Patterned Surface Charge
}

\author{
Yi-Kuen Lee, Member, IEEE, Lap Man Lee, Winky Lap Wing Hau, and Yitshak Zohar
}

\begin{abstract}
The technology developed for photolithographically patterning the electric surface charge to be negative, positive, or neutral enables the realization of complex liquid flows even in straight and uniform microchannels with extremely small Reynolds number. A theoretical model to analyze a steady incompressible electrokinetically driven two-dimensional liquid flow in a microchannel with an inhomogeneous surface charge under externally applied electric field is derived. The flow field is obtained analytically by solving the biharmonic equation with the Helmholtz-Smoluchowski slip boundary condition using the Fourier series expansion method. The model has been applied to study three basic out-of-plane vortical flow fields: single vortex and a train of corotating and a series of counterrotating vortex pairs. For model verification, the solution for the single vortex has been tested against numerical computations based on the full Navier-Stokes equations revealing the dominant control parameters. Two interesting phenomena have been observed in out-of-plane multivortex dynamics: merging of corotating vortices and splitting of counterrotating vortices. The criteria for the onset of both phenomena are discussed.

[2006-0114]
\end{abstract}

Index Terms-Electrokinetic effect, microchannel, microfluidics, surface charge pattern, vortex.

\section{INTRODUCTION}

$\mathbf{M}$ ICROFLUIDIC systems have attracted major research interest due to promising potential applications in biotechnology, in particular in micro total analysis systems or the lab-on-a-chip. Typically, an assay carried out in such a microsystem involves flow of buffer solutions, reaction, separation, and detection [1]-[3]. Hence, the control of liquid flow is an integral element in the operation of such fluidic microsystems. Pressure and gravity are typically the forces applied to drive liquid flows in macrochannels. However, when the characteristic length scale of the channel is too small, surface forces acting on the flow (e.g., friction) become dominant in comparison to body forces (e.g., inertia). Consequently, flows driven by surface forces, such as electroosmosis, are recently receiving great attention for controlling liquid motion in microchannels [4]. Electroosmotic flow not only is more efficient as the channel size decreases but also requires no moving parts.

Manuscript received June 21, 2006; revised August 14, 2006. This work was supported by the Hong Kong Research Grants Council under RGC Grants HKUST6017/02E and 616106. Subject Editor C.-J. Kim.

Y.-K. Lee and W. L. W. Hau are with the Department of Mechanical Engineering, Hong Kong University of Science and Technology, Clear Water Bay, Kowloon, Hong Kong, China (e-mail: meyklee@ust.hk).

L. M. Lee and Y. Zohar are with the Department of Aerospace and Mechanical Engineering, University of Arizona, Tucson, AZ 85721-00119 USA.

Digital Object Identifier 10.1109/JMEMS.2006.885992
Thus, the fabrication process of microsystems incorporating electroosmosis flow is much simpler.

Solid surfaces are likely to carry electrostatic charge, i.e., an electric surface potential, due to broken bonds and surface charge traps [5]. When an electrolyte solution is brought into contact with such a solid boundary, the surface charge attracts the counter-ions in the liquid establishing an electric field. The arrangement of the electrostatic charges on the solid surface and the balancing charges in the liquid is called the electric double layer (EDL) [6]. Counter-ions are strongly attracted to the surface forming an immobile compact layer at the solid/liquid interface. Outside this layer, the distribution of the counter-ions away from the interface decays within the diffuse layer with a characteristic thickness known as the Debye length-scale, which is inversely proportional to the square root of the liquid ionic concentration. The electric potential at the boundary between the compact and diffuse layers is called zeta potential (or $\zeta$ potential). Thus, a channel with walls having fixed charges generates a mobile "sheath" of ions in the fluid. Under an externally applied electric field parallel to a surface with net charge density, the mobile ions move in a direction parallel to the wall. The movement of this sheath induces the motion of the bulk liquid, due to shear stress, as if the walls of the channel were sliding at a velocity directly proportional to both the magnitude of the applied field and the mobility of the ions. The ion mobility is a function of the surface charge density, liquid ionic strength, liquid viscosity, and permittivity.

Anderson and Idol [7] demonstrated, for the first time, that the liquid flow field inside a circular microchannel (pore) with nonuniform charged walls can have multiple vortices due to electroosmosis. Most of the studies have focused on electroosmotic flows with externally applied uniform electric field and uniform zeta potential along a straight microchannel. For example, Dutta and Beskok solved combined electroosmotic/pressure driven flows in two-dimensional (2-D) straight channels [8]. However, the charge distribution in the microchannel does not have to be uniform. Ajdari demonstrated that a simple uniform electric field can generate transverse fluid currents on inhomogeneously charged surfaces [9]. Qian and Bau argued that chaotic advection is possible when the zeta potential along the channel walls is modulated spatially and temporally [10], [11]. Hau et al. showed that dramatically enhanced mixing rate can be obtained by utilizing inhomogeneous surface charge pattern [12].

A variety of techniques have been proposed to locally generate various charge patterns. Stroock et al. used laminar flow to generate longitudinal patterns, while a modified version of micromolding in capillaries was used to create transverse patterns 


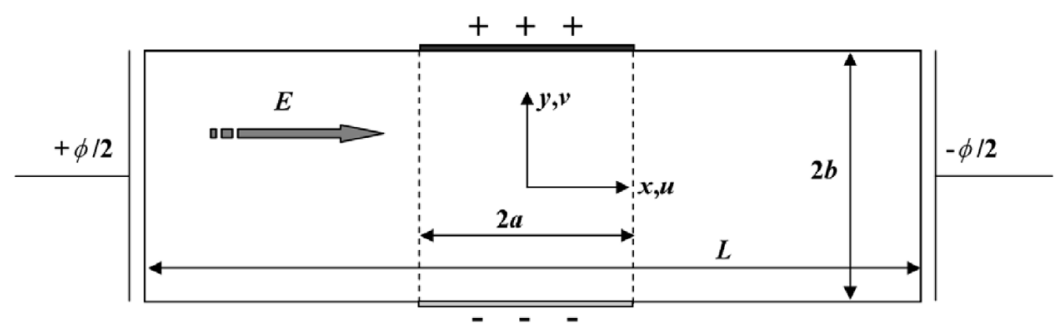

Fig. 1. A schematic illustration of the surface charge pattern for generating a single out-of-plane vortex in a microchannel.

[13]. In another approach, Hau et al. used polymer coating by electrostatic self-assembly (ESA) combined with standard photolithography techniques to selectively define the surface charge pattern [14]. Using this technology, a series of both out-of-plane [15] and in-plane vortices [16] has been demonstrated in microchannel liquid flow. However, due to various constraints in the experiments, all the findings are limited to qualitative analysis, while the details of the flow fields have not been explored. For theoretical formulation of the flow field in a rectangular cavity with the nonuniform $\zeta$ potential, Qian and Bau derived a two-dimensional time-independent and time-dependent model to show that there is a chaotic flow by using time-wise periodic modulation of the $\zeta$ potential [10], [11]. In this paper, a biharmonic equation is solved with the Helmholtz-Smoluchowski slip boundary condition [11], [20] to study electrokinetically driven out-of-plane vortical liquid flow in a straight and uniform microchannel with different surface-charge patterns, including single vortex, corotating vortices and its merging, counterrotating vortices, and vortex splitting.

\section{OUt-OF-Plane Single VorteX}

The surface-charge pattern for generating a single out-of-plane spanwise vortex consists of a positively or negatively charged region on either the top or bottom channel surface with the corresponding region on the opposite surface carrying the opposite charge [18]. The active area is placed at the center, while the rest of the microchannel surface is kept neutral. The countervelocity slip, induced by a streamwise electric field near the top and bottom surfaces, results in a recirculation zone as shown in Fig. 1, where $x$ is the streamwise and $y$ the cross-stream coordinate. $L$ and $b$ are the length and half-height of the microchannel, respectively, while $a$ is half of the active-region length.

\section{A. Theoretical Analysis}

For two-dimensional low Reynolds number flow $(\operatorname{Re} \rightarrow 0)$, the incompressible Navier-Stokes equation can be simplified into a two-dimensional biharmonic equation [19]

$$
\nabla^{4} \Psi(x, y)=0
$$

where $\Psi$ is the stream function, with the streamwise $u$ and crossstream velocity component, $v$ calculated as

$$
u=\frac{\partial \Psi}{\partial y}, \quad v=-\frac{\partial \Psi}{\partial x}
$$

It is reasonable to assume that there is a slip velocity at the channel wall if the channel hydraulic diameter $D_{h}$ (typically $10 \mu \mathrm{m}$ ) is very much larger than the EDL thickness $\lambda_{\mathrm{D}}$ (typically $100 \mathrm{~nm}$ ), i.e., $\lambda_{\mathrm{D}} / \mathrm{D}_{\mathrm{h}} \ll 1$. In addition, we assume that the $\zeta$ potential at the boundary between positively and negatively charged surfaces changes in a discrete manner, which is a good approximation in the cases where the nonuniformity of the $\zeta$ potential is confined to a very small region compared with the microchannel size. Thus, in this paper, the electrokinetic effect is modeled as a velocity slip imposed at the charged channel wall. The magnitude of the velocity slip $q$ is given by the Helmholtz-Smoluchowski equation [20]

$$
q=\left|\frac{E \varepsilon \zeta}{\mu}\right|=\left|\frac{\phi \varepsilon \zeta}{\mu L}\right|
$$

The externally applied electric field $E$ along a uniform microchannel can be calculated by dividing the imposed potential difference $\phi$ by the distance $L$ separating the positive and negative electrodes. The final solution is then obtained by reducing the governing equation (1) to an ordinary differential equation using the separation of variables and imposing the nonuniform velocity slip boundary condition using Fourier series expansion. Referring to Fig. 1, the velocity slip boundary conditions are imposed discretely at the active regions on the microchannel top and bottom surfaces as follows:

$$
\begin{array}{r}
u(x,-b)=\frac{\partial \Psi(x,-b)}{\partial y}= \begin{cases}q, & \text { for }|x| \leq a \\
0, & \text { for }|x|>a\end{cases} \\
u(x, b)=\frac{\partial \Psi(x, b)}{\partial y}= \begin{cases}-q, & \text { for }|x| \leq a \\
0, & \text { for }|x|>a\end{cases}
\end{array}
$$

along with the impermeability condition at all microchannel solid surfaces

$$
v(x, \pm b)=-\frac{\partial \Psi(x, \pm b)}{\partial x}=0 .
$$

Note that the periodic flow boundary conditions in the $x$ direction are assumed at the left and right ends of the microchannel.

To mathematically represent the boundary conditions on the top and the bottom walls along $x$-direction, Joseph and Sturges calculated the series coefficients by using a biorthogonal series to construct the infinite set of algebraic equations, namely, Papkovich-Fadle eigenfunctions [21]. Meleshko applied the method of superposition with series acceleration to provide a better accuracy with a relatively small number of terms in 
Fourier series [22], [23], which was also adopted by Qian and Bau [10], [11]. In this paper, nevertheless, we use the basic Fourier series expansion because it is very simple and accurate enough if the number of terms is determined properly with the help of numerical simulation. As the boundary conditions are even functions in the $x$ direction, the solution can be solved using the Fourier series expansion method. The solution can be assumed to be a series solution as follows:

$$
\Psi(x, y)=\sum_{i}^{\infty} \psi(x, y)=\sum_{i}^{\infty} \cos (\alpha x) f(y), \quad \alpha \equiv \frac{i \pi}{L}
$$

where $f(y)$ is the function to be determined and $i$ is the number of terms used in the Fourier series. It is assumed that the channel is long enough that the boundary conditions on the walls at both ends do not disturb the flow field. This assumption can be verified by examining the velocity solutions at the end walls.

The governing equation (1) can now be reduced to a fourthorder ordinary differential equation for $f(y)$

$$
\frac{d^{4} f(y)}{d y^{4}}-2 \alpha^{2} \frac{d^{2} f(y)}{d y^{4}}+\alpha^{4} f(y)=0
$$

which has the following general solution:

$$
\begin{aligned}
f(y)=c_{1} \cosh (\alpha y)+ & c_{2} \sinh (\alpha y) \\
& +c_{3} y \cosh (\alpha y)+c_{4} y \sinh (\alpha y)
\end{aligned}
$$

where $c_{1}, c_{2}, c_{3}$, and $c_{4}$ are coefficients to be determined from the boundary conditions. In order to obtain the expression for the velocity boundary conditions, we find

$$
\begin{aligned}
f^{\prime}(y)= & c_{1} \alpha \sinh (\alpha y)+c_{2} \alpha \cosh (\alpha y) \\
& +c_{3}(\alpha y \sinh (\alpha y)+\cosh (\alpha y)) \\
& +c_{4}(\alpha y \cosh (\alpha y)+\sinh (\alpha y)) .
\end{aligned}
$$

Using (7), the boundary conditions [(4) and (5)] can be simplified into

$$
\frac{\partial \Psi(x, \pm b)}{\partial x}=-\alpha \sin (\alpha x) f( \pm b)=0
$$

resulting in

$$
f(-b)=f(b)=0
$$

and

$$
\frac{\partial \Psi(x, \pm b)}{\partial y}=\cos (\alpha x) f^{\prime}( \pm b)
$$

which can be expressed using the Fourier series expansion

$$
\begin{gathered}
\cos (\alpha x) f^{\prime}(-b)=\frac{q a}{L}+\sum_{n=1}^{\infty} A_{n} \cos (\alpha x) \\
A_{n}=\frac{2 q \sin (\alpha a)}{n \pi}
\end{gathered}
$$

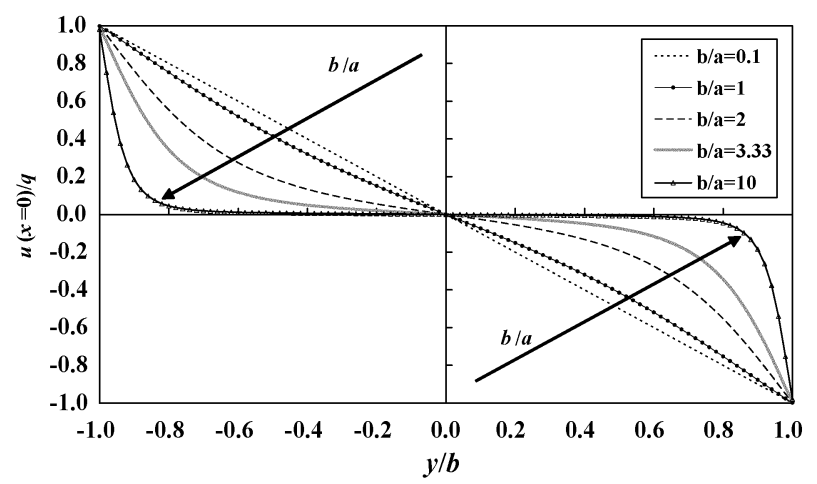

(a)

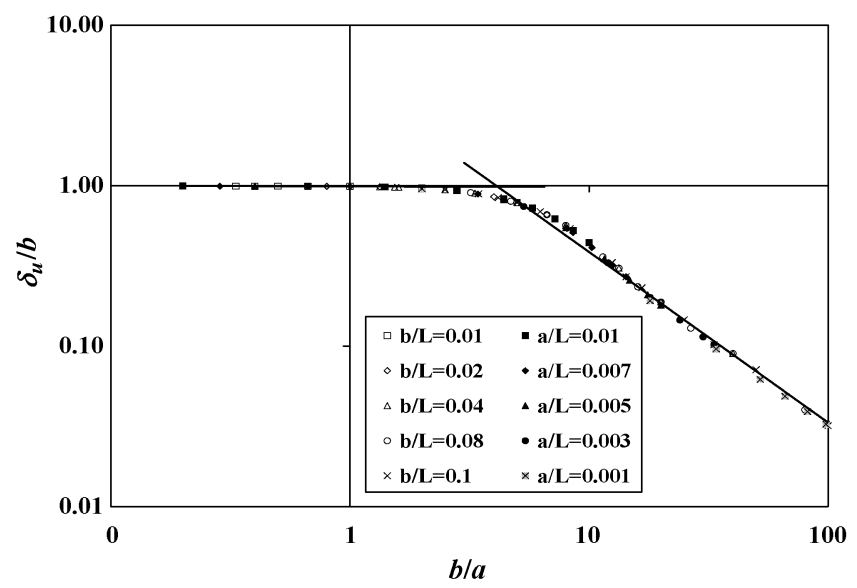

(b)

Fig. 2. The effect of the $b / a$ ratio on (a) the streamwise velocity profiles $u(y)$ across the channel center $(x=0)$ and (b) the velocity-decay length scale $\delta_{u}$ for a single out-of-plane vortex.

$$
\begin{gathered}
\cos (\alpha x) f^{\prime}(b)=-\frac{q a}{L}+\sum_{n=1}^{\infty} B_{n} \cos (\alpha x) \\
B_{n}=\frac{-2 q \sin (\alpha a)}{n \pi} .
\end{gathered}
$$

The four coefficients $c_{1}, c_{2}, c_{3}$, and $c_{4}$ can now be determined by substituting the four boundary conditions (12) and (14) into (9) and (10) as follows:

$$
\begin{aligned}
c_{1} & =2 A_{n} \frac{b \sinh (\alpha b)}{2 a b+\sinh (2 \alpha b)} \\
& =\frac{4 q \sin (\alpha a)}{n \pi} \frac{b \sinh (\alpha b)}{2 \alpha b+\sinh (2 \alpha b)} \\
c_{2} & =-\left(A_{n}+B_{n}\right) \frac{b \cosh (\alpha b)}{-2 \alpha b+\sinh (2 a b)}=0 \\
c_{3} & =\left(A_{n}+B_{n}\right) \frac{\sinh (\alpha b)}{-2 \alpha b+\sinh (2 a b)}=0 \\
c_{4} & =2 A_{n} \frac{\cosh (\alpha b)}{2 a b+\sinh (2 \alpha b)} \\
& =-\frac{4 q \sin (\alpha a)}{n \pi} \frac{\cosh (\alpha b)}{2 \alpha b+\sinh (2 \alpha b)} .
\end{aligned}
$$




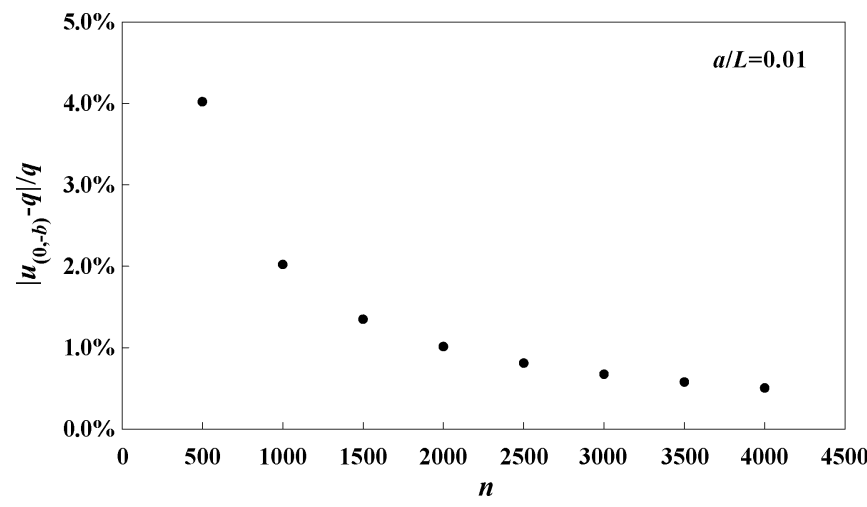

Fig. 3. Convergence test of the analytical solution using the relative error of wall velocity at the center of surface charge region $x=0$ and $y=-b$ as a function of the number of expansion terms.

Substitution of the coefficients in (15) into (7) yields

$$
\Psi(x, y)=\psi_{0}(x, y)+\sum_{n=1}^{\infty} \psi_{n}(x, y)
$$

with

$$
\psi_{0}(x, y)=\frac{a q}{L} \frac{b^{2}-y^{2}}{2 b}
$$

and

$$
\begin{aligned}
& \psi_{n}(x, y)=\cos (\alpha x) \frac{4 q \sin (\alpha a)}{n \pi} \\
& \quad \times\left(\frac{b \sinh (\alpha b) \cosh (\alpha y)-y \cosh (\alpha b) \sinh (\alpha y)}{2 \alpha b+\sinh (2 \alpha b)}\right) .
\end{aligned}
$$

Thus, the stream function is given by (18) as shown at the bottom of the page and the velocity components $u$ and $v$ are readily available, as shown in (19) at the bottom of the page. By substituting the velocity solution (19) into the boundary conditions (4)-(6), the boundary condition (6) can be exactly satisfied. The root-mean-square (rms) error (calculated by means of integration) in the evaluation of the boundary conditions (4) and (5) using (19) was found to be less than $1 \%$ when the number of terms was chosen to be larger than 100 . However, the solution

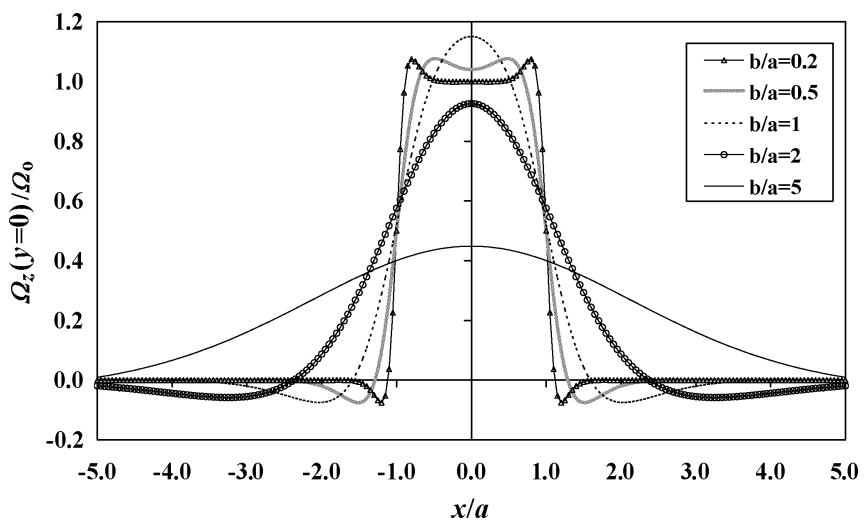

Fig. 4. Spanwise vorticity profile of a single out-of-plane vortex along the channel mid-height $y=0$ with $b / a$ ratio as the varying parameter.

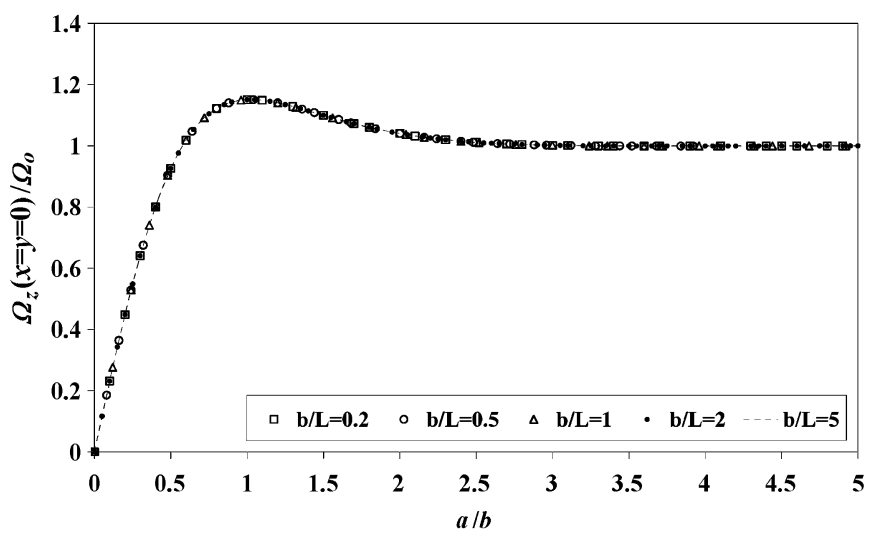

Fig. 5. Dependence of the single out-of-plane vortex core vorticity $\Omega_{z}(x=$ $y=0$ ) on the ratio $b / a$.

(19) around the singular points described in the boundary conditions of (4)-(6) can have an overshoot much larger than the rms error due to Gibbs phenomenon. Away from these singular points, (19) is accurate enough to describe the vortex flow pattern.For a given electric field and working fluid, where $q=$ $|\mathrm{E} \varepsilon \zeta / \mu|$ is fixed, the velocity field depends only on the channel height $2 b$ and the active-region length $2 a$. Equation (18) suggests that either increasing $b$ or decreasing $a$ will have a similar effect. Therefore, the transverse profile of the streamwise

$$
\Psi(x, y)=\frac{q a\left(b^{2}-y^{2}\right)}{2 b L}+\sum_{n=1}^{\infty} \frac{4 q \cos (\alpha x) \sin (\alpha a)(b \cosh (\alpha y) \sinh (\alpha b)-y \cosh (\alpha b) \sinh (\alpha y))}{n \pi(2 \alpha b+\sinh (2 \alpha b))}
$$

$$
\begin{aligned}
& u(x, y)=-\frac{q a y}{b L}+\sum_{n=1}^{\infty} \frac{4 q \cos (\alpha x) \sin (\alpha a)[\alpha b \sinh (\alpha y) \sinh (\alpha b)-\cosh (\alpha b) \sinh (\alpha y)-\alpha y \cosh (\alpha b) \cosh (\alpha y)]}{n \pi(2 \alpha b+\sinh (2 \alpha b))} \\
& v(x, y)=\sum_{n=1}^{\infty} \frac{4 q \sin (\alpha x) \sin (\alpha a)(b \cosh (\alpha y) \sinh (\alpha b)-y \cosh (\alpha b) \sinh (\alpha y))}{L(2 \alpha b+\sinh (2 \alpha b))}
\end{aligned}
$$




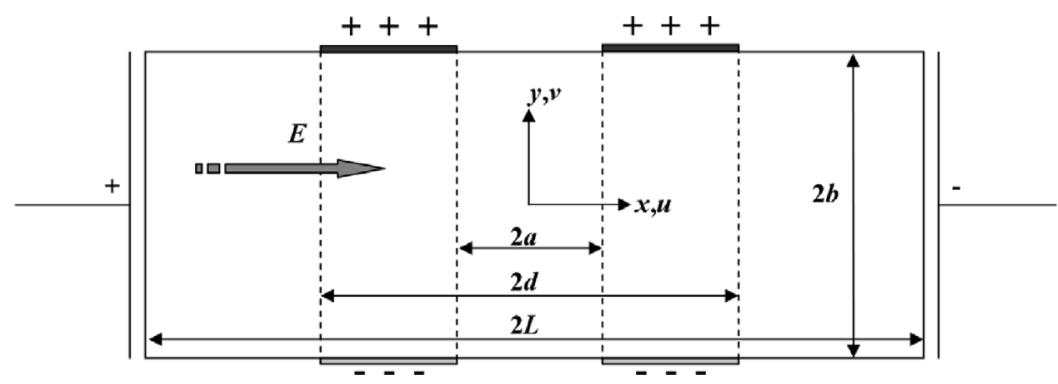

Fig. 6. A schematic illustration of the surface charge pattern for generating a pair of out-of-plane corotating vortices in a microchannel.

velocity $u$ at the channel center $x=0$ is plotted in Fig. 2(a) for several values of the ratio $b / a$. The velocity profile is linear for smaller values $b / a<1$, similar to Couette flow between two plates moving in opposite directions. As the ratio increases, the velocity profile becomes highly nonlinear. For large values $(b / a>5)$ the velocity of the bulk of the fluid away from the walls approaches zero.

The velocity at the center of the active region is selected for the convergence criterion and is plotted in Fig. 3 as a function of the number of expansion terms. The number of terms used for the analytical solution is determined such that the calculated velocity is within $1 \%$ of the prescribed velocity $q$. However, the convergence of the center velocity depends also on the length of the active region. Thus, the test has been repeated for every configuration discussed in this paper and the number of terms used in the calculations adjusted to ensure the accuracy of the velocity and vorticity solution.

\section{B. Flow Field Characterization}

In order to characterize the decay of $u$ away from the channel wall, a length scale $\delta_{u}$, similar to the boundary layer thickness in the boundary layer theory [19] and the electric double layer thickness [8], is defined as the distance from the wall at which the streamwise velocity drops to $1 \%$ of its maximum level $q . \delta_{u} / b$ is plotted as a function of $b / a$ in Fig. 2(b). At the lower range, $b / a<1$, the active-region length compared to the channel height is large enough to allow momentum transfer from the walls to the middle of the channel resulting in the linear velocity profile. Hence, $\delta_{u} / b=0.98$ is independent of the ratio $b / a$ over this lower range. However, for the higher range $(b / a>5)$, the curve drops with a constant slope in the logarithmic plot as the ratio $b / a$ increases. In this case, the active region is too small compared to the height, and the momentum generated near the walls cannot be transferred to the midchannel. The flow region near the walls with finite velocity, as indicated by $\delta_{u} / b$, decreases with increasing $b / a$, while the velocity of the flow interior away from the walls diminishes to zero.

In the 2-D flow under investigation, only the spanwise component of the vorticity exists, i.e., $\Omega_{z}(x, y) \equiv$ $\partial v / \partial x-\partial u / \partial y \neq 0$. The total circulation along the channel walls and channel ends is the integral of the spanwise vorticity in the channel given by

$$
\Gamma=\int_{-b}^{b} \int_{-L / 2}^{L / 2} \Omega z d x d y=4 a q
$$

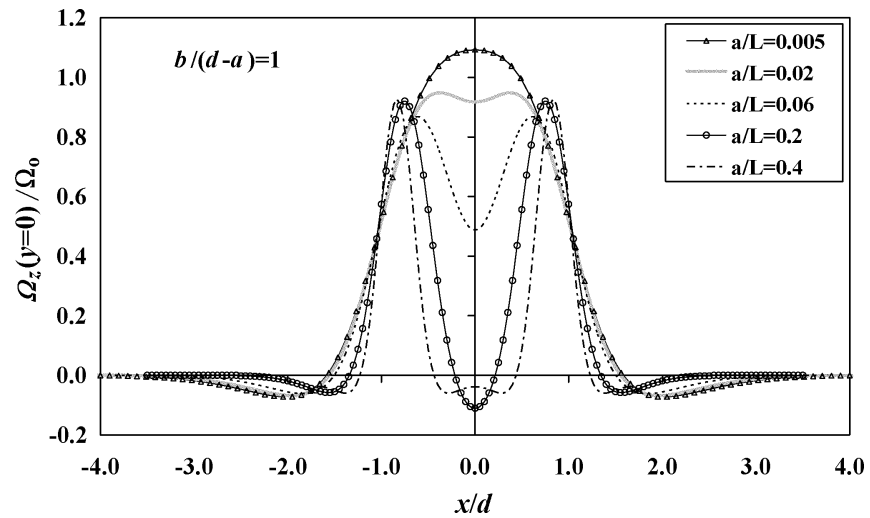

Fig. 7. Streamwise profiles of spanwise vorticity $\Omega_{z}$ along the channel midheight $(y=0)$ for the pair of out-of-plane corotating vortices with the ratio $a / L$ as the varying parameter.

which is a constant dictated by the boundary conditions, i.e., linearly proportional to the active-region length and the slip velocity at the wall. The vorticity profile along the channel midheight $\Omega_{z}(x, y)=0$, extracted in Fig. 4, illustrates the vorticity associated with the single out-of-plane vortex. The vorticity profile indicates both the location of the vortex core, at $x=y=0$, and the vortex strength.

The normalized vorticity at the vortex center $\Omega_{z}(0,0) /(q / b)$ is plotted in Fig. 5 as a function of $a / b$. Both components $\partial u / \partial y$ and $\partial v / \partial x$ continuously drop in magnitude when $a / b$ is decreasing; the vorticity vanishes at the limit $b \gg a$ (i.e., $a / b \rightarrow$ 0 ). On the other hand, for $a>b$, as the velocity profile $u(y)$ becomes linear such that the vorticity is dominated by $|\partial u / \partial y| \sim$ $q / b$, the normalized vorticity at the center asymptotically approaches unity. From Figs. 6 and 7, it is clear that there exists one optimal $b / a$ ratio $(=1)$ for generation of maximum vorticity $\Omega_{z}$. For the value of $b / a$ ratio less than one and larger than one, the vorticity $\Omega_{z}$ will be smaller. Note that in most practical microchannels, the microchannel length $L$ is usually much larger than the microchannel height $(2 b)$.

\section{CoRotating Out-of-Plane Vortices}

A train of corotating out-of-plane vortices can be formed by periodic duplication of the charge pattern required for a single vortex as sketched in Fig. 6. Note that in this section half of the channel length is denoted by $L$ for convenience. Half the separation between the active regions is denoted by $a$, while $d$ is half the distance between the far edges of two neighboring active regions; thus, the active length is equal to $(d-a)$. 


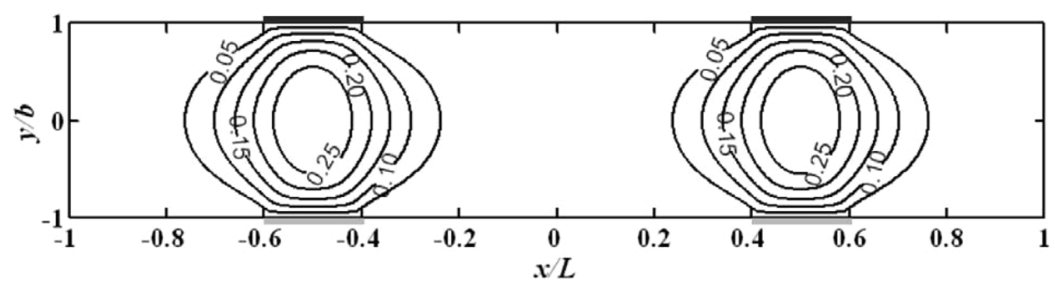

(a)

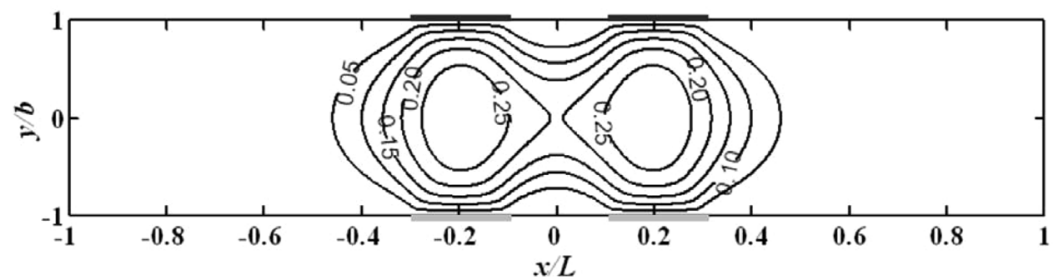

(b)

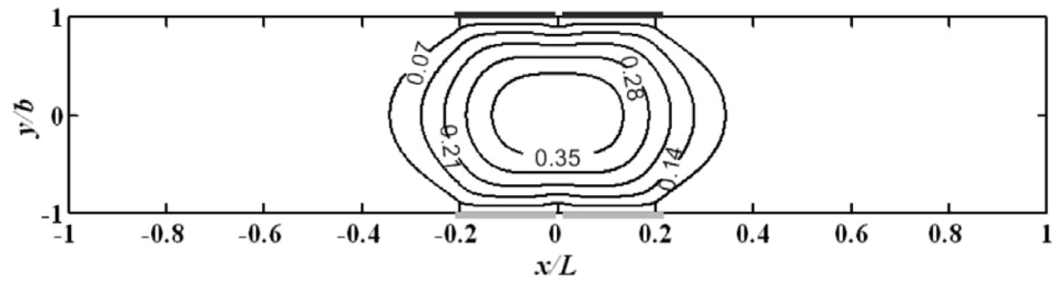

(c)

Fig. 8. Contour plots of streamlines demonstrating the merging of out-of-plane corotating vortices with decreasing $a / L$ ratio: (a) $a / L=0.4$, (b) $a / L=0.1$, and (c) $a / L=0.01$ for the case of $b /(d-a)=1$.

\section{A. Analytical Derivation}

For simplicity, only a pair of corotating vortices in a channel of finite length $L$ is considered, as it is straightforward to extend the solution to a larger number of vortices. The main interest is placed on the domain between the active regions $(|x|<d)$ to investigate the interaction between neighboring vortices. Similar to the single vortex case, the slip and impermeability boundary conditions are imposed as follows:

$$
\begin{aligned}
u(x,-b) & =\frac{\partial \Psi(x,-b)}{\partial y} \\
& = \begin{cases}0, & \text { for } 0 \leq|x|<a \text { and } d \leq|x|<L \\
q, & \text { for } a \leq|x|<d\end{cases} \\
u(x, b) & =\frac{\partial \Psi(x, b)}{\partial y} \\
& = \begin{cases}0, & \text { for } 0 \leq|x|<a \text { and } d \leq|x|<L \\
-q, & \text { for } a \leq|x|<d\end{cases} \\
v(x, \pm b) & =-\frac{\partial \psi(x, \pm b)}{\partial x}=0 .
\end{aligned}
$$

Assuming the solution to be in the form

$$
\Psi(x, y)=\sum_{i}^{\infty} \psi(x, y)=\sum_{i}^{\infty} \cos (\alpha x) f(y), \quad \alpha \equiv \frac{i \pi}{L}
$$

and expanding the boundary conditions along the top and bottom walls using Fourier series

$$
\begin{array}{r}
\frac{\partial \psi(x,-b)}{\partial y}=\frac{q(d-a)}{L}+\sum_{n=1}^{\infty} A_{n} \cos (\alpha x) \\
A_{n}=\frac{2 q[\sin (\alpha d)-\sin (\alpha a)]}{n \pi} \\
\frac{\partial \psi(x, b)}{\partial y}=-\frac{q(d-a)}{L}+\sum_{n=1}^{\infty} B_{n} \cos (\alpha x) \\
B_{n}=-\frac{2 q[\sin (\alpha d)-\sin (\alpha a)]}{n \pi} .
\end{array}
$$

The solution of the stream function is found to be

$$
\begin{aligned}
\Psi(x, y) \\
=\frac{q(d-a)\left(b^{2}-y^{2}\right)}{2 b L} \\
+\sum_{n=1}^{\infty} \frac{4 q \cos (\alpha x)[\sin (\alpha d)-\sin (\alpha a)]}{n \pi} \\
\quad \times\left[\frac{b \sinh (\alpha b) \cosh (\alpha y)-y \cosh (\alpha b) \sinh (\alpha y)}{(2 \alpha b+\sinh (2 \alpha b))}\right]
\end{aligned}
$$

with the velocity components $u$ and $v$ given by (28) as shown at the bottom of the next page.

\section{B. Vortex Merging}

Spanwise vorticity profiles along the channel mid-height $\Omega_{z}(x, y=0)$ are calculated as shown in Fig. 7, for the particular 


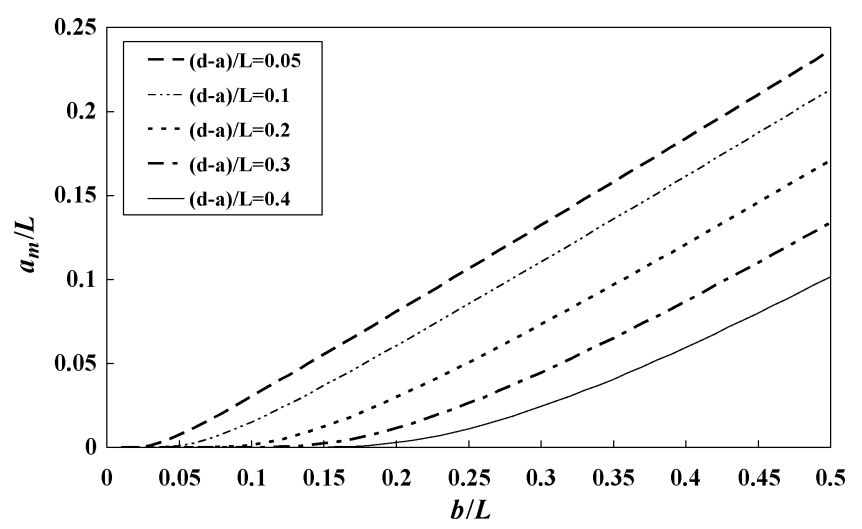

Fig. 9. The effect of channel height and active-region length on the onset of merging of corotating vortices: the vortex cells are separated above and are merged below each curve with of $(d-a) / L$ as the varying parameter.

configuration of $b /(d-a)=1$ with the normalized active-region length $a / L$ as the varying parameter. When the separation between the active regions is sufficiently large $(a / L>0.2)$, both the velocity $v$ and the velocity gradient $\partial v / \partial x$ vanish at the channel center $(x=0)$. Accordingly, two peaks of the same height are clearly distinguished in the vorticity profile. This indicates the development of distinct corotating vortices, as observed in the streamline plot in Fig. 8(a), with little interaction between them. As the separation between the active regions decreases $(a / L<0.2)$, the two vortices start to interact with each other, as shown in Fig. 8(b), although the vortex cores are still distinct. For very small separation $(a / L<0.02)$, the cross-stream velocity profile becomes monotonic, accompanied by a single peak in the vorticity profile, indicating the complete merging of the two corotating vortices into a single one as shown in Fig. 8(c). Using this as a criterion, the conditions for the emergence of a single vortex can be estimated. The critical distance between the two active regions, denoted by $a_{m}$, depends on both the channel height $2 b$ and the active-region length $(d-a)$. The results are summarized in Fig. 9, where the critical distance increases with either increasing channel height or decreasing active-region length. Clearly, for vanishing

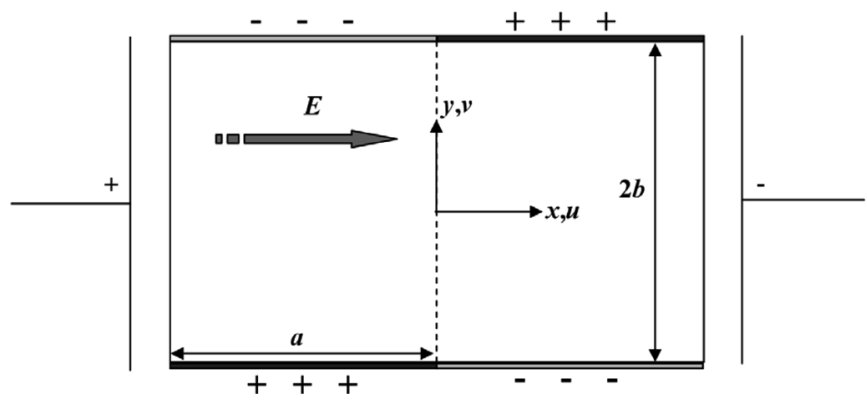

Fig. 10. A schematic illustration of the surface charge pattern for generating periodic pairs of out-of-plane counter-rotating vortices in an infinitely long microchannel.

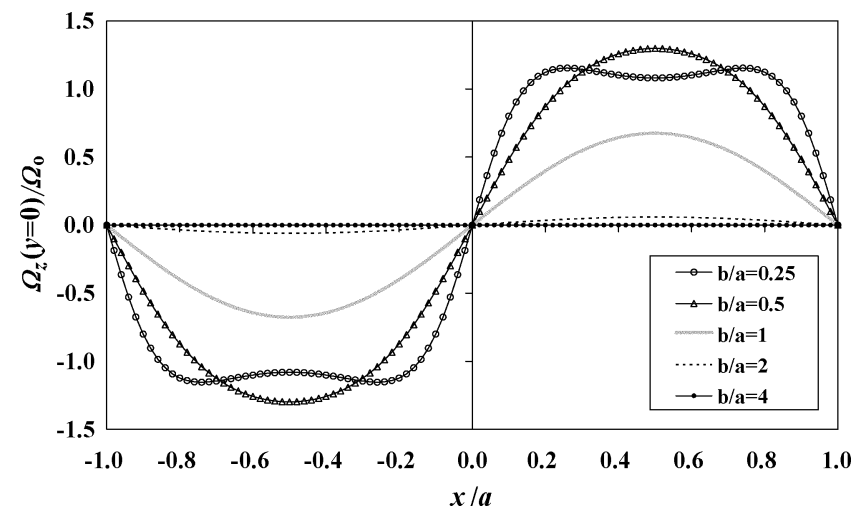

Fig. 11. Streamwise profiles of spanwise vorticity $\Omega_{z}$ along the channel midheight $(y=0)$ for the pair of out-of-plane counterrotating vortices with the ratio $b / a$ as the varying parameter.

separation distance, i.e., $a=0$, the single-vortex solution discussed in the previous section is recovered.

\section{Counterrotating Out-OF-Plane Vortices}

Counterrotating pairs of out-of-plane vortices periodically distributed along the channel can also be formed by flipping the charge pattern in every alternate cell in the configuration used for the corotating vortices as illustrated in Fig. 10. Here, $a$ represents the active-region length such that one wavelength is $2 a$.

$$
\begin{aligned}
u(x, y)= & -\frac{q(d-a) y}{b L}+\sum_{n=1}^{\infty} \frac{4 q \cos (\alpha x)[\sin (\alpha d)-\sin (\alpha a)]}{n \pi} \\
& \times\left[\frac{\alpha b \sinh (\alpha b) \sinh (\alpha y)-\cosh (\alpha b) \sinh (\alpha y)-\alpha y \cosh (\alpha b) \cosh (\alpha y)}{(2 \alpha b+\sinh (2 \alpha b))}\right] \\
v(x, y)= & \sum_{n=1}^{\infty} 4 q \sin (\alpha x)[\sin (\alpha d)-\sin (\alpha a)] \\
& \times\left[\frac{b \sinh (\alpha b) \cosh (\alpha y)-y \cosh (\alpha b) \sinh (\alpha y)}{L(2 \alpha b+\sinh (2 \alpha b))}\right]
\end{aligned}
$$




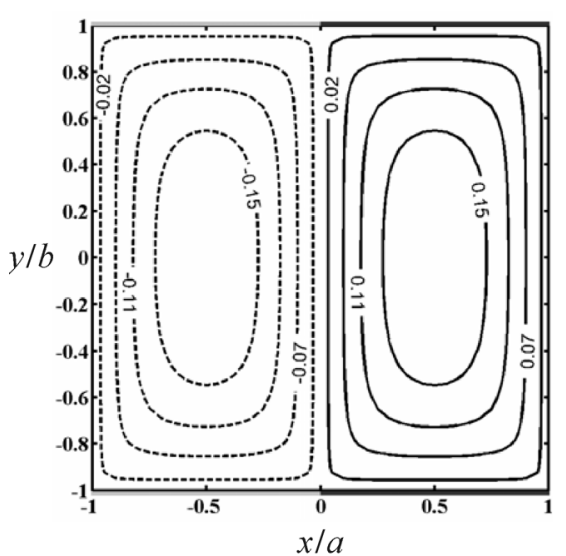

(a)

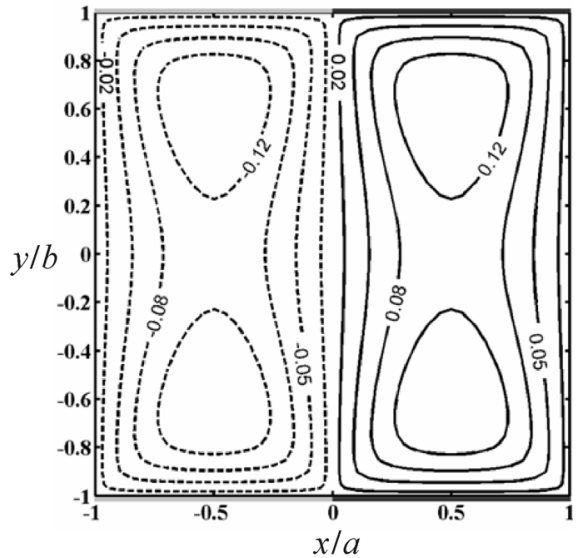

(b)

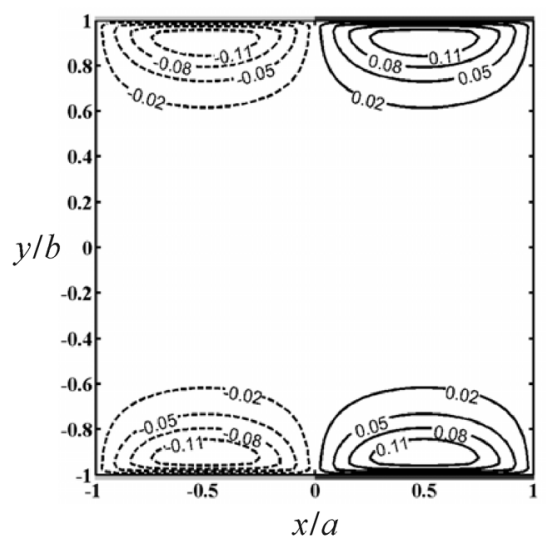

(c)

Fig. 12. Contour plots of streamlines demonstrating the splitting of out-of-plane counterrotating vortices with increasing $b / a$ ratio: (a) $b / a=0.5$, (b) $b / a=1.0$, and (c) $b / a=4.0$.

\section{A. Analytical Derivation}

One pair of counterrotating vortices is considered, as it can easily be extended to a large number of pairs along the channel. The imposed boundary conditions are

$$
\begin{gathered}
u(x,-b)=\frac{\partial \Psi(x,-b)}{\partial y}= \begin{cases}q, & \text { for } 0 \leq x<a \\
-q, & \text { for }-a \leq x<0\end{cases} \\
u(x, b)=\frac{\partial \Psi(x, b)}{\partial y}= \begin{cases}-q, & \text { for } 0 \leq x<a \\
q, & \text { for }-a \leq x<0\end{cases} \\
v(x, \pm b)=-\frac{\partial \Psi(x, \pm b)}{\partial x}=0 .
\end{gathered}
$$

The slip boundary condition is an odd function in $x$-direction; hence, the solution is in the form

$$
\Psi(x, y)=\sum_{i}^{\infty} \psi(x, y)=\sum_{i}^{\infty} \sin (\alpha x) f(y), \quad \alpha \equiv \frac{i \pi}{L}
$$

Using the following Fourier series expansions:

$$
\begin{aligned}
& \frac{\partial \Psi(x,-b)}{\partial y}= \sum_{n=1}^{\infty} A_{n} \sin (\alpha x) \\
& A_{n}=\frac{2 q\left(1-(-1)^{n}\right)}{n \pi}
\end{aligned}
$$

$$
\begin{array}{r}
\frac{\partial \Psi(x, b)}{\partial y}=\sum_{n=1}^{\infty} B_{n} \sin (\alpha x) \\
B_{n}=\frac{-2 q\left(1-(-1)^{n}\right)}{n \pi}
\end{array}
$$

the stream function is calculated as follows:

$$
\begin{aligned}
& \Psi(x, y)=-\sum_{n=1}^{\infty} 4 q \sin (\alpha x) \frac{\left(-1+(-1)^{n}\right)}{n \pi} \\
& \times\left[\frac{b \sinh (\alpha b) \cosh (\alpha y)-y \cosh (\alpha b) \sinh (\alpha y)}{(2 \alpha b+\sinh (2 \alpha b))}\right]
\end{aligned}
$$

and the velocity components $u$ and $v$ are given by (35) and (36) as shown at the top of the next page.

\section{B. Vortex Splitting}

Spanwise vorticity profiles along the channel mid-height $\Omega_{z}(x, y=0)$ are calculated as shown in Fig. 11 with the ratio $b / a$ as the varying parameter. When the channel height is sufficiently small compared with the active-region length $(b / a<0.5)$, the peak values in the symmetric velocity and antisymmetric vorticity profiles remain the same. These profiles indicate the existence of a pair of counterrotating vortices as observed in the streamline plot in Fig. 12(a). As the channel height becomes comparable to the active-region length $(b / a \sim 1)$, the magnitude of both the velocity and vorticity associated with 


$$
\begin{aligned}
u(x, y)= & -\sum_{n=1}^{\infty} 4 q \sin (\alpha x) \frac{\left(-1+(-1)^{n}\right)}{n \pi} \\
& \times\left[\frac{\alpha b \sinh (\alpha b) \sinh (\alpha y)-\cosh (\alpha b) \sinh (\alpha y)-\alpha y \cosh (\alpha b) \cosh (\alpha y)}{(2 \alpha b+\sinh (2 \alpha b))}\right] \\
v(x, y)= & \sum_{n=1}^{\infty} 4 q\left(-1+(-1)^{n}\right) \cos (\alpha x) \\
& \times\left[\frac{b \sinh (\alpha b) \cosh (\alpha y)-y \cosh (\alpha b) \sinh (\alpha y)}{a(2 \alpha b+\sinh (2 \alpha b))}\right]
\end{aligned}
$$

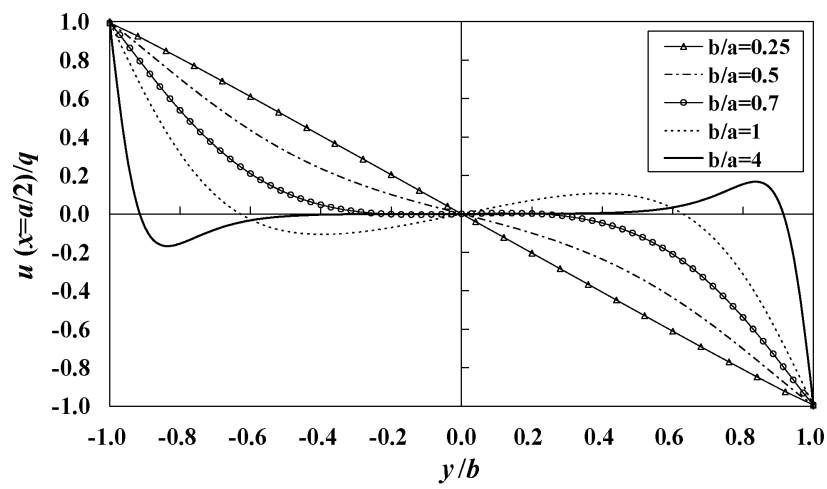

(a)

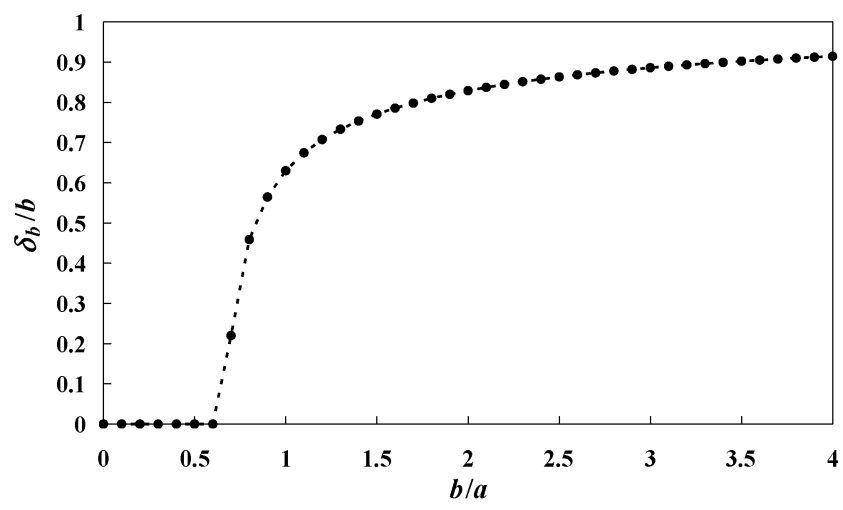

(b)

Fig. 13. The effect of channel height and active-region length on the onset of splitting of counterrotating vortices: (a) streamwise velocity profiles $u(y)$ across an active-region center $(x=a / 2)$ and (b) the back-flow length scale $\delta_{b}$ as a function of $b / a$ ratio.

each vortex decreases. This marks the start of a pinchoff process where two vortex cores with the same sense of rotation develop within each active region, as shown in Fig. 12(b). As the ratio increases further $(b / a>2)$, both the cross-stream velocity and the spanwise vorticity vanish at the channel mid-height, indicating a complete split of each single vortex into a pair of distinct vortices with the same sense of rotation, as shown in Fig. 12(c). A sharper criterion for the onset of the vortex splitting condition can be inferred from the streamwise velocity profiles across the vortex core shown in Fig. 13(a). For large $b / a$ ratio, a region of counterflow appears above and below the channel mid-height corresponding to the emergence of two vortex cores. The extent of this region, denoted by $\delta_{b}$, is plotted as a function of $b / a$ in Fig. 13(b). The condition for vortex splitting is then $\delta_{b}>0$ when $b / a>0.6$.

\section{CONCLUSIONS}

Complex liquid flow fields can be generated in a microchannel, although the Reynolds number is very low, by applying electric field along the channel while taking advantage of patterned surface charge on the channel walls. A theoretical model to analyze a steady incompressible electrokinetically driven two-dimensional microchannel liquid flow is obtained by solving the biharmonic equation with Helmholtz-Smoluchowski slip boundary condition. The model has been applied to study three basic out-of-plane vortical flow fields: single vortex and a train of corotating and a series of counterrotating vortex pairs.

For the single out-of-plane vortex configuration, the effect of increasing the channel height is found to be similar to decreasing the active-region length, suggesting that the ratio between these two length scales is the proper control parameter for this flow. When the ratio is smaller than unity, the streamwise velocity profile across the vortex core resembles that of Couette flow, while the cross-stream velocity profile is similar to that of a viscous vortex. In corotating vortices, vortex merging takes place when the separation distance between the active regions is sufficiently small compared to the channel height and, at the limit of vanishing separation distance, the single-vortex solution is recovered. On the other hand, in the series of counterrotating out-of-plane vortices, vortex splitting occurs for sufficiently large channel height to active-region length ratio, i.e., larger than 0.6.

\section{REFERENCES}

[1] M. A. Burn et al., "An integrated nanoliter DNA analysis device," Science, vol. 282, pp. 484-487, Oct. 1998.

[2] D. R. Reyes, D. Iossifidis, P. A. Auroux, and A. Manz, "Micrototal analysis systems: 1. Introduction, theory, and technology," Anal. Chem., vol. 74, pp. 2623-2636, Jun. 2002.

[3] P. A. Auroux, D. Iossifidis, D. R. Reyes, and A. Manz, "Micrototal analysis systems: 2. Anaytical standard operations and application," Anal. Chem., vol. 74, pp. 2637-2652, Jun. 2002.

[4] L. Bousse et al., "Electrokinetically controlled microfluidic analysis systems," Annu. Rev. Bioph. Biom., vol. 29, pp. 155-182, 2000.

[5] P. Bertrand, A. Jonas, A. Laschewsky, and R. Legras, "Ultrathin polymer coatings by complexation of polyelectrolytes at interfaces: Suitable materials, structure and properties," Macromol. Rapid Commun., vol. 21, pp. 319-348, Apr. 2000.

[6] R. J. Hunter, Zeta Potential in Colloid Science: Principles and Applications. London, U.K.: Academic, 1981. 
[7] J. L. Anderson and W. K. Idol, "Electroosmosis through pores with nonuniformly charged walls," Chem. Eng. Commun., vol. 38, pp. 93-106, 1985.

[8] P. Dutta and A. Beskok, "Analytical solution of combined electroosmotic/pressure driven flows in two-dimensional straight channels: Finite Debye layer effects," Anal. Chem., vol. 73, pp. 1979-1986, May 2001.

[9] A. Ajdari, "Electro-osmosis on inhomogeneously charged surfaces," Phys. Rev. Lett., vol. 75, pp. 755-758, Jul. 1995.

[10] S. Qian and H. H. Bau, "A chaotic electoosmotic stirrer," Anal. Chem., vol. 74, pp. 3616-3625, Aug. 2002.

[11] _ - "Theoretical investigation of electro-osmotic flows and chaotic stirring in rectangular cavities," Appl. Math. Model., vol. 29, pp. 726-753, Aug. 2005.

[12] W. L. W. Hau, L. M. Lee, Y.-K. Lee, and Y.Zohar, "Electrically driven vortical motion for mixing of liquids in a microchannel," in Proc. MicroTAS 2003, Squaw Valley, CA, Oct. 5-9, 2003, pp. 491-494.

[13] A. D. Stroock, M. Weck, D. T. Chiu, W. T. S. Huck, P. J. A. Kenis, R. F. Ismagilov, and G. M. Whitesides, "Patterning electro-osmotic flow with patterned surface charge," Phys. Rev. Lett., vol. 84, pp. 3314-3317, Apr. 2000.

[14] W. L. W. Hau, D. W. Trau, N. J. Sucher, M. Wong, and Y. Zohar, "Surface-chemistry technology for microfluidics," J. Micromech. Microeng., vol. 13, pp. 272-278, Mar. 2003.

[15] _ "Micro flow patterns on demand using surface-chemistry technology," in Proc. MEMS 2002, Las Vegas, NV, Jan. 20-24, 2002, pp. 475-478.

[16] W. L. Hau, L. M. Lee, Y.-K. Lee, M. Wong, and Y. Zohar, "Experimental investigation of electrokinetically generated in-plane vorticity in a microchannel," in Proc. Transducers 2003, Boston, MA, Jun. 9-12, 2003, pp. 651-654.

[17] A. Ajdari, "Generation of transverse fluid currents and forces by an electric field: Electro-osmosis on charge-modulated and undulated surfaces," Phys. Rev. E, vol. 53, pp. 4996-5005, May 1996.

[18] A. S. W. Ng, W. L. W. Hau, Y.-K. Lee, and Y. Zohar, "Electrokinetic generation of microvortex patterns in a microchannel liquid flow," $J$. Micromech. Microengng., vol. 14, pp. 247-255, Feb. 2004.

[19] H. Schlichting, Boundary-Layer Theory. New York: McGraw-Hill, 1979.

[20] R. F. Probstein, Physiochemical Hydrodynamics: An Introduction. Boston, MA: Butterworths, 1989.

[21] D. D. Joseph and L. D. Sturges, "The convergence of biorthogonal series for biharmonic and stokes flow edge problems: Part II," SIAM J. Appl. Math., vol. 34, pp. 7-26, Jan. 1978.

[22] V. V. Meleshko, "Steady stokes flow in a rectangular cavity," Proc. $R$. Soc. Lond. A, vol. 452, pp. 1999-2022, Sep. 1996.

[23] V. V. Meleshko and A. M. Gomilko, "Infinite systems for a biharmonic problem in a rectangular," Proc. R. Soc. Lond. A, vol. 453, pp. 2139-2160, Oct. 1997.

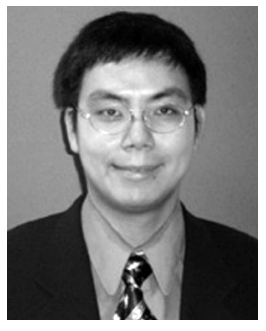

Yi-Kuen Lee (S'97-M'02) received the B.S. degree (with honors) in agricultural machinery engineering (currently bioindustrial mechantronics engineering) and the M.S. degree in applied mechanics from the National Taiwan University, Taipei, Taiwan, R.O.C., and the $\mathrm{Ph} . \mathrm{D}$. degree in mechanical engineering (with the microelectromechanical systems major) from University of California, Los Angeles, in 2001.

$\mathrm{He}$ is currently an Assistant Professor in the Department of Mechanical Engineering at the Hong Kong University of Science and Technology. His research interests include bio-MEMS, micro/nanofluidics, micro electroporation and electrofusion, micro/nano electrophoresis, and micro/nano heat transfer.

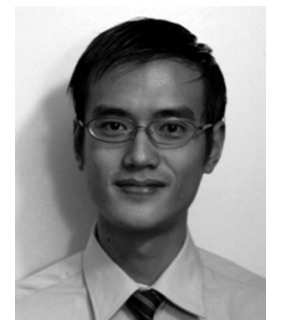

Lap Man Lee graduated (with first-class honors) from the Department of Mechanical Engineering, the Hong Kong University of Science and Technology. He then moved to the University of Arizona, Tucson, and received the M.Sc. degree in aerospace and mechanical engineering. Currently, he is working toward the Ph.D. degree in the Bioengineering Option at the California Institute of Technology, Pasadena.

His research interests include optofluidic integration, biomedical imaging devices, and microfluidics.

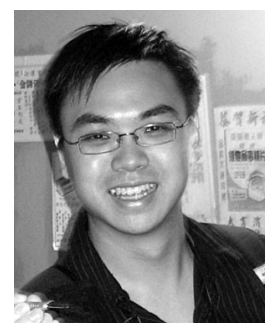

Winky Lap Wing Hau received the B.Eng. degree in mechanical engineering from the Hong Kong University of Science and Technology in 1998 Subsequently, he received the M.Phil. degree in the electronic packaging research group, lead by Prof. Matthew Yuen in the same department, focusing on stress-sensing chip, in 2001. He then pursued his field of interest in MEMS and joined Prof. Yitshak Zohar's group to receive the Ph.D. degree from the same department in 2005.

He took the leading role in establishing experimental foundations of electro-osmoic flow research in the group. His Ph.D research work was published in several leading conferences and journals in MEMS. Currently, he contiunes to pursue fundamental research on hydrodynamic interactions between spheres and Stokes flow in microchannels at the Institute of Microsystem Technology (IMTEK), the University of Freiburg, Germany. He works in the MEMS Applications group, lead by Prof. Roland Zengerle.

Dr. Hau is a Research Fellow of The Croucher Foundation, Hong Kong, which provides full financial support for his research in IMTEK.

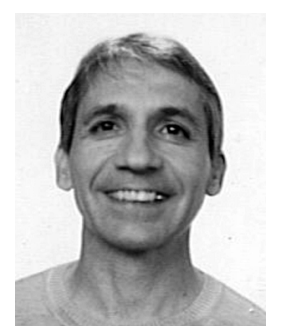

Yitshak Zohar received the B.S. and M.S. degrees in aeronautical engineering from the Technion-Israel Institute of Technology, Haifa, in 1981 and 1984, respectively. He received the Ph.D. degree in aerospace engineering from the University of Southern California (USC), Los Angeles, in 1990.

As a Research Associate at USC and the University of California, Los Angeles (UCLA) (1990-1992), he started to work on microelectromechanical systems. He joined the Department of Mechanical Engineering at the Hong Kong University of Science and Technology as one of the founding faculty members in 1992, where he participated in setting up the University Micro Fabrication Center and established the Department Micromachines Laboratory. At the end of 2003, he took up a Professor position in the Aerospace and Mechanical Engineering Department at the University of Arizona, Tucson, to develop a MEMS/Bio-MEMS program and to establish a Micro/Nano Fabrication Center. His research interests are the science and technology of microsystems and, in particular, microscale fluid mechanics and heat transfer. Recently, he has started to work on Bio-MEMS subjects such as microcapillary electrophoresis, patterning multiproteins, and binding kinetics of particles/cells with derivatized surfaces.

Dr. Zohar is a Fellow of the American Society of Mechanical Engineers (ASME). 